Draft Version OCtOBER 12, 2018

Preprint typeset using LATEX style emulateapj v. 6/22/04

\title{
COSMIC TRANSIENTS TEST EINSTEIN'S EQUIVALENCE PRINCIPLE OUT TO GEV ENERGIES
}

\author{
He $\mathrm{GaO}^{1,2,3,4,6}$, Xue-Feng Wu ${ }^{1,2,5}$, Peter Mészáros ${ }^{2,3,4}$ \\ ${ }^{1}$ Purple Mountain Observatory, Chinese Academy of Sciences, Nanjing 210008, China; xfwu@pmo.ac.cn \\ ${ }^{2}$ Department of Astronomy and Astrophysics, Pennsylvania State University, 525 Davey Laboratory, University Park, PA 16802: \\ ${ }^{3}$ Department of Physics, Pennsylvania State University, 525 Davey Laboratory, University Park, PA 16802 \\ ${ }^{4}$ Center for Particle and Gravitational Astrophysics, Institute for Gravitation and the Cosmos, Pennsylvania State University, 525 Davey \\ Laboratory, University Park, PA 16802 \\ ${ }^{5}$ Joint Center for Nuclear Particle Physics and Cosmology, Purple Mountain Observatory - Nanjing University, Nanjing 210008, China \\ ${ }^{6}$ Department of Astronomy, Beijing Normal University, Beijing 100875, China \\ Draft version October 12, 2018
}

\begin{abstract}
The Einstein Equivalence Principle (EEP) can be probed with astrophysical sources emitting simultaneously different types of neutral particles, or particles with varying energies, by testing their time of flight through the same gravitational field. Here we use the time delays between correlated photons from cosmological transients to constrain the accuracy of the EEP. We take data from two gammaray bursts as an example, and use, as a lower limit to the theoretical time delays between different energies, delays arising from only the gravitational field of our own galaxy. We then show that the parameterized post-Newtonian parameter $\gamma$ is the same for photons over energy ranges between $\mathrm{eV}$ and $\mathrm{MeV}$ and between $\mathrm{MeV}$ and $\mathrm{GeV}$ to a part in $10^{-7}$, which is at least one order of magnitude better than previous limits. Combining this bound on the wavelength dependence of $\gamma$ with the absolute bound $|\gamma-1|<0.3 \%$ from light-deflection measurements at optical $(\mathrm{eV})$ wavelengths, we thus extend this absolute bound on $\gamma$ to $\mathrm{GeV}$ energies.

Subject headings:
\end{abstract}

\section{INTRODUCTION}

One statement of the Einstein Equivalence Principle (EEP) is that any uncharged test body traveling in empty space will follow a trajectory independent of its internal structure and composition. For test bodies with macroscale masses, the accuracy of the EEP can be measured in a Newtonian context. However, for test particles like photons and neutrinos, Newtonian dynamics is no longer precise enough. To test the accuracy of the EEP at this level, at least the lowest-order deviations from Newton's law of universal gravitation are required, which can be conveniently described by the parameterized post-Newtonian (PPN) formalism. For all metric theories of gravitation in which all bodies satisfy the EEP, the PPN formalism explicitly details the parameters in which a general theory of gravity can differ from Newtonian gravity. Each theory is specified by a set of numerical coefficients (PPN parameters) see (Will 2006, 2014, for a review).

A gravity theory can be tested through the absolute values of PPN parameters. For example, it has been pointed out that the time interval required for photons to traverse a given distance is longer in the presence of a gravitational potential $U(r)$ by

$$
\delta t=-\frac{1+\gamma}{c^{3}} \int_{r_{e}}^{r_{o}} U(r) d r
$$

where $r_{e}$ denotes the location of the source of particle emission and $r_{o}$ that of observation (Shapiro 1964; Krauss \& Tremaine 1988; Longo 1988). Half of the delay is caused by the warping of space by the gravitational field, the other half can be thought of as a result of gravitational redshift. Here $\gamma$ is one of the PPN parameter, representing how much space curvature is produced by unit rest mass. Different gravity theories predict differ- ent values for $\gamma$. For instance, general relativity predicts that $\gamma=1$ (Will 1993). Many precise methods have been devised to test the accuracy of general relativity through the measurement of the value of $\gamma$, the most precise being the measurement of the gravitational deflection of light near the Sun and the round-trip travel time delay of an artificial radar signal due to the solar system gravity (Will 2006, 2014):

- the most precise results from the deflection of light is through very-long-baseline radio interferometry measurement, yielding $\gamma-1=(-0.8 \pm 1.2) \times$ $10^{-4}$ (Lambert \& Le Poncin-Lafitte 2009, 2011);

- the most precise results from the travel time delay of a radar signal, which is also the most precise constraints on the $\gamma$ value to date, is from the Doppler tracking of the Cassini spacecraft, yielding $\gamma-1=(2.1 \pm 2.3) \times 10^{-5}$ (Bertotti et al. 2003).

It is worth pointing out that $\gamma=1$ is not a sufficient condition to identify general relativity, since it is not the only theory that predicts $\gamma=1$ (Will 1993). Regardless of the absolute value of $\gamma$, all metric theories of gravity that incorporate the EEP predict that all test particles (photons or neutrinos) must follow identical trajectories and undergo the same time delay, even though the delay time may vary between different theories. In other words, as long as the EEP is valid, all metric theories predict $\gamma=\gamma_{1}=\gamma_{2}$ (and $\gamma$ need not even be 1 ), where 1 and 2 represent two different test particles. In this case, the accuracy of the EEP can be characterized by limits on the differences of $\gamma$ value for different species of particles, or the same specie of particle with varying energies.

It has been proposed that the close coincidence in time of arrival of the photon and neutrino bursts from the supernova 1987A provides a strong test of the EEP (Longo 
1988; Krauss \& Tremaine 1988). Comparing the arrival time delay of the photons and neutrinos, Longo (1988) proposed that the $\gamma$ values for photon $(\mathrm{eV})$ and neutrino $(\mathrm{MeV})$ are identical to an accuracy of approximately $0.37 \%$. On the other hand, the very small dispersion in arrival times observed for neutrinos of different energies $(7.5-40 \mathrm{MeV})$ can be used to set a limit on the energy dependence of $\gamma_{\nu}$ to the level of $1.6 \times 10^{-6}$.

Supernova 1987A is an extragalactic source, but noncosmological. In this work, we propose that the time delays between correlated photons from cosmological transients, such as Gamma-Ray Bursts (GRBs), can also be used to constrain the EEP with similar approaches. In the following, we first discuss the general picture of using cosmic transients to constrain the EEP. Taking GRBs as an example, we then show the ability of this method for improving the constraints, and discuss the uncertainties involved.

\section{GENERAL DESCRIPTION FOR DERIVING EEP CONSTRAINTS WITH COSMIC TRANSIENTS}

Cosmic transient sources often show some time delays between different energy bands. The main terms that might contribute to this time delay may be expressed as follows:

$$
\Delta t_{\mathrm{obs}}=\Delta t_{\mathrm{int}}+\Delta t_{\mathrm{LIV}}+\Delta t_{\mathrm{spe}}+\Delta t_{\mathrm{gra}}
$$

In the following, we discuss each component in turn.

- $\Delta t_{\text {int }}$ is the intrinsic (astrophysical) time delay between two test photons, and this has the largest uncertainty since it is impossible to determine it based on the observational data alone. Appropriate assumptions for the radiation mechanism are usually required to assign a chronological order for the different energy photons, but it is still hard to estimate the exact value of $\Delta t_{\text {int }}$.

- $\Delta t_{\mathrm{LIV}}$ represents the potential time delay due to Lorentz invariance violation (LIV) via an energydependent velocity of light. Some descriptions of it are based on quantum fluctuations in the background space-time metric causing a non-trivial dispersion in vacuo for light propagating through this space-time foam (Amelino-Camelia et al. 1998). Taking into account the effect on the propagation of photons due to the expansion of the Universe, even a tiny variation in photon speed may lead to an observable time delay (Ellis et al. 2006; Abdo et al. 2009),

$$
\Delta t_{L I V}=\frac{(1+n)}{2 H_{0}} \frac{E_{1}^{n}-E_{2}^{n}}{E_{Q G, n}^{n}} \int_{0}^{z} \frac{\left(1+z^{\prime}\right)^{n} d z^{\prime}}{h\left(z^{\prime}\right)},
$$

which is the n-th (first non-vanishing term) of a series expansion in an effective field theory formulation of quantum gravity including Lorentz invariance violating terms. Here $E_{1}$ and $E_{2}$ represent the energies for two test photons $\left(E_{1}>E_{2}\right), E_{Q G}$ is the effective energy scale of quantum gravity, $h(z)=H(z) / H_{0}$, with $H_{0}$ being the present Hubble expansion rate and $H(z)$ the Hubble parameter at different redshifts. For a limiting $E_{Q G}$ equal to the Planck energy, the recent evidence disfavors the existence of linear (order $n=1$ ) LIV terms, i.e. energy-dependent departures of the speed of photons from the usual speed of light (Ellis et al. 2006; Abdo et al. 2009; Pan et al. 2015). Substituting the Plank energy for $E_{Q G}$ it is easy to show that the higher order terms of the expansion $(n>2)$, even if they exist, are negligible for the purposes of this work ${ }^{1}$. We thus ignore this term in the following analysis.

- $\Delta t_{\text {spe }}$ represents the potential time delay due to special-relativistic effects in the case where the photons have a rest mass which is non-zero, which reads

$\Delta t_{\mathrm{spe}}=\frac{m_{\mathrm{ph}}^{2} c^{4}}{2 H_{0}}\left(\frac{1}{E_{1}^{2}}-\frac{1}{E_{2}^{2}}\right) \int_{0}^{z} \frac{\left(1+z^{\prime}\right) d z^{\prime}}{h\left(z^{\prime}\right)}$,

where $m_{\mathrm{ph}}$ is the photon rest mass. Modern experiments have provided the upper limits for the photon rest mass as $m_{\mathrm{ph}}<10^{-18} \mathrm{eV} / \mathrm{c}^{2}$ Amsler et al. 2008). In this case, $\Delta t_{\text {spe }}$ is negligible even when the energy of test photons are lower than radio band.

- $\Delta t_{\text {gra }}$ corresponds to the difference in arrival time of two photons of energy $E_{1}$ and $E_{2}$, caused by the gravitational potential $U(r)$ integrated from the emission site to the Earth, which can be estimated with equation 1, as long as the expression of $U(r)$ is known,

$$
\Delta t_{\text {gra }}=\frac{\gamma_{1}-\gamma_{2}}{c^{3}} \int_{r_{o}}^{r_{e}} U(r) d r
$$

where $c=3 \times 10^{10} \mathrm{~cm} \mathrm{~s}^{-1}$ is the energyindependent speed for massless particles. In general, $U(r)$ can be divided into three parts: the gravitational potential of our galaxy $U_{\mathrm{mw}}(r)$ would be dominant out to a certain distance, say to the edge of the local group $(\sim 1 \mathrm{Mpc})$. Based on the cosmological principle and current CMB results, the gravitational potential should gradually approach a flat background intergalactic potential $U_{\mathrm{IG}}(r)$ between our galaxy and the host galaxy (or cluster) of the transient. This assumption may be valid at least for sources with $z<2$, for which the lensing probability is significant only to $<1 \%$ (Wiklind et al. 2013). The final contribution is from the gravity well of the host galaxy (or the relevant galaxy cluster) $U_{\text {host }}(r)$.

Leaving out the negligible components, equation 2 is

$$
\Delta t_{\text {obs }}=\Delta t_{\text {int }}+\Delta t_{\text {gra }}
$$

For a specific case where high energy photons arrive later than the low energy photons $\left(\Delta t_{\mathrm{obs}}>0\right)$, if we know that $\Delta t_{\text {int }}>0$, we will have

$$
\Delta t_{\mathrm{obs}}>\frac{\gamma_{1}-\gamma_{2}}{c^{3}} \int_{r_{o}}^{r_{e}} U(r) d r
$$

The potential function for $U_{\mathrm{IG}}(r)$ and $U_{\text {host }}(r)$ is hard to model, but it is very likely that the effect of these two

\footnotetext{
${ }^{1}$ In general, one can find that as long as $E_{Q G, n} / E_{1} \gg 10^{18 / n}$, the $n$-th expansion of $\Delta t_{L I V}$ becomes negligible for the purposes of this work (e.g., much smaller than the order of a second).
} 
terms is much larger than if we simply assumed that the potential is just $U_{\mathrm{mw}}(r)$ extended to the distance of the transient, i.e.,

$$
\Delta t_{\mathrm{obs}}>\frac{\gamma_{1}-\gamma_{2}}{c^{3}} \int_{r_{o}}^{r_{e}} U_{\mathrm{mw}}(r) d r
$$

Although the gravitational potential of the Milky Way at large distances is still not well known, Krauss \& Tremaine (1988) examined two popular potential models, e.g., the Keplerian potential $U(r)=-G M / r$ and the isothermal potential $U(r)=v_{c}^{2}\left[\ln \left(r / r_{\max }\right)-1\right]$, for $r<r_{\max }$, where $v_{c}$ is the circular speed at the solar radius and the potential becomes Keplerian for $r>r_{\max }=100 \mathrm{kpc}$. They proposed that different models for $U_{\mathrm{mw}}(r)$ do not have a strong influence on the results for testing EEP. In this work, we adopt the Keplerian potential for our galaxy. Thus, for the purposes of obtaining a lower limit, we can simply extend this galactic potential out to cosmic scales to bracket from below the potential function of $U_{\mathrm{IG}}(r)$ and $U_{\text {host }}(r)$. We thus have

$$
\gamma_{1}-\gamma_{2}<\Delta t_{\mathrm{obs}}\left(\frac{G M_{\mathrm{mw}}}{c^{3}}\right)^{-1} \ln ^{-1}\left(\frac{d}{b}\right)
$$

where $G=6.68 \times 10^{-8} \mathrm{erg} \mathrm{cm} \mathrm{g}^{-2}$ is the gravitational constant, $M_{\mathrm{mw}} \approx 6 \times 10^{11} M_{\odot}$ is the mass of our galaxy (McMillan 2011; Kafle et al. 2012), $d$ is the distance from the transient to Earth, and $b$ is the impact parameter of the light rays relative to the center of the galaxy. For a cosmic source in the direction $\left(\mathrm{R} . \mathrm{A} .=\beta_{S}\right.$, Dec. $\left.=\delta_{S}\right)$, the impact parameter can be estimated as

$b=r_{G} \sqrt{1-\left(\sin \delta_{S} \sin \delta_{G}+\cos \delta_{S} \cos \delta_{G} \cos \left(\beta_{S}-\beta_{G}\right)\right)^{2}}$

where $r_{G}=8.3 \mathrm{kpc}$ is the distance from the Sun to the galaxy center, and $\left(\beta_{G}=17^{\mathrm{h}} 45^{\mathrm{m}} 40.04^{\mathrm{s}}, \quad \delta_{G}=\right.$ $\left.-29^{\circ} 00^{\prime} 28.1^{\prime \prime}\right)$ are the coordinates of the galaxy center in the equatorial coordinate system (J2000; Gillessen et al. 2009). Note that a more accurate formulation for $U_{\mathrm{mw}}(r)$ may provide higher order corrections to equation 9 , but the correction would be absorbed into the approximation obtained from replacing $U_{\mathrm{IG}}(r)$ and $U_{\text {host }}(r)$ with the extension of $U_{\mathrm{mw}}(r)$.

\section{EEP CONSTRAINTS WITH GRBS}

Gamma-ray bursts are the most extreme explosive events in the universe. They were initially observed as short, intense, and non-repeating flashes of $\sim \mathrm{MeV} \gamma$ rays. Later on, it was found that the initial burst is usually followed by a longer-lived broadband emission (X-ray, ultraviolet, optical, infrared, microwave and radio), showing that GRBs are actually multi-wavelength transients, instead of simply bursts of $\gamma$-rays. The initial $\mathrm{MeV} \gamma$-ray emission is often called the "prompt emission, while the longer wavelength emission is referred to as the "afterglow emission. It is worth noticing that in some bursts, the longer wavelength emission, e.g. optical emission, sometimes already starts during the prompt emission phase. The duration of the prompt emission is relatively short, ranging from $\sim 0.1 \mathrm{~s}$ to $\sim 1000 \mathrm{~s}$. Morever, the histogram of the duration shows a bimodal structure separated at $2 \mathrm{~s}$, where bursts with duration less than $2 \mathrm{~s}$ are classified as short-GRBs and those that last for more than $2 \mathrm{~s}$ are called long- GRBs. (Kumar \& Zhang 2014, for a review).

GRBs are interesting cosmic transients for obtaining EEP constraints for the following reasons:

- Even the $\gamma$-ray photons are usually observed in different energy bands, for instance, the two instruments onboard Fermi, the Gamma-ray Burst Monitor (GBM; Meegan et al. (2009)) and the Large Area Telescope (LAT; Atwood et al. (2009)), provide unprecedented spectral coverage for seven orders of magnitude in energy (from $\sim 8 \mathrm{keV}$ to $\sim 300$ $\mathrm{GeV})$.

- The observed $\gamma$-ray lightcurves in each energy band often have violent variability, with minimum timescales as small as milliseconds (Bhat et al. 1992). It is easy to use these sharp features to identify the arrival time lag between different energy photons.

- Thanks to the prompt slewing capability of the XRay Telescope (XRT; Burrows et al. (2005)) and the UV-Optical Telescope (UVOT; Roming et al. $(2005))$ onboard the Swift satellite (Gehrels et al. 2004), longer wavelength photons have been observed at very early times after the GRB trigger, even during the prompt emission phase. It is thus possible to determine the arrival lag between $\gamma$-ray photons and low energy photons, such as optical photons.

After four decades of observation, thousands of GRBs have been studied in significant detail, making it possible to find some good examples that can be used to constrain the EEP. In this work, we take two examples, GRB 090510 and GRB 080319B.

\subsection{GRB 090510}

GRB 090510 first triggered the Fermi-GBM on a precursor at $T_{0}=00: 22: 59.97$ UT, May 10th, 2009, and the main emission episode in the $8 \mathrm{keV}-40 \mathrm{MeV}$ energy range started $\sim 0.5 \mathrm{~s}$ after $T_{0}$, lasting up to $\sim 1$ s. Then, $0.43 \mathrm{~s}$ after the GBM trigger, it was detected and precisely localized by the Swift satellite, with coordinates (J2000) R.A. $=22^{\mathrm{h}} 14^{\mathrm{m}} 12.47^{\mathrm{s}}$, Dec. $=$ $-26^{\circ} 35^{\prime} 00.4^{\prime \prime}$ (Hoversten et al. 2009). The emission observed by the Fermi- LAT started $0.65 \mathrm{~s}$ after the GBM trigger and lasted $\sim 200 \mathrm{~s}$ (Guiriec et al. 2009; Ohno \& Pelassa 2009). The $T_{90}$ of the burst is $0.3 \mathrm{~s}$, placing it in the short GRB category. Subsequent observations determined its redshift as $z=0.903 \pm 0.003$ (Rau et al. 2009; McBreen et al. 2010).

Abdo et al. (2009) analyzed in detail its prompt light curve over the different energy bands observed by Fermi. They found that the total GBM light curve $(8 \mathrm{keV}-$ $40 \mathrm{MeV}$ ) consists of seven main pulses, where the first one is a dim short spike. A single $31-\mathrm{GeV}$ photon was detected by LAT at $0.83 \mathrm{~s}$ after the GBM trigger, which coincides in time with the last of the seven GBM pulses. The directional and temporal coincidence of this photon with the $\mathrm{MeV}$ radiation of GRB 090510 is very significant, at the $>5 \sigma$ confidence level (Abdo et al. 2009). No high-energy $(\mathrm{GeV})$ photon has ever been detected before the onset of the low-energy $(\mathrm{MeV})$ emission in a GRB, 
and this $31-\mathrm{GeV}$ photon similarly appears likely to have been emitted after the onset of GRB 090510. Taking as our nominal time delay $\Delta t \simeq 0.83 \mathrm{~s}$, we thus have from eq. (9) for this burst

$$
\gamma_{\mathrm{GeV}}-\gamma_{\mathrm{MeV}}<2 \times 10^{-8}
$$

\subsection{GRB 080319B}

GRB 080319B was detected by Swift at $T_{0}=$ 06 : 12 : 49 UT, March 19th, 2008, with coordinates $(\mathrm{J} 2000)$ R.A. $=14^{\mathrm{h}} 31^{\mathrm{m}} 40.7^{\mathrm{s}}$, Dec. $=+36^{\circ} 18^{\prime} 14.7^{\prime \prime}$ (Racusin et al. 2008a). It is a long burst with $T_{90}>50 \mathrm{~s}$ and located at $z=0.937$ (Vreeswijk et al. 2008). At the trigger time of this burst, the wide-field robotic optical telescope "Pi of the Sky" (Cwiok et al. 2007) and the wide- field robotic instrument Telescopio Ottimizzato per la Ricerca dei Transienti Ottici Rapidi (TORTORA; Molinari et al. (2006)) both serendipitously had the GRB within their fields of view (as they were both already observing GRB 080319A). A bright optical transient was observed starting at $2.75 \pm 5 \mathrm{~s}$ and peaking around $18 \mathrm{~s}$ after the Swift trigger. The peak V-band magnitude reached 5.3, corresponding to a flux density of $\sim 28.7$ Jy (Karpov et al. 2008; Zou et al. 2009). TORTORA measured the brightest portion of the optical flash with high time resolution, distinguishing three separate peaks which enabled doing detailed comparisons between the prompt optical and $\gamma$-ray emissions. Kumar \& Narayan (2009) calculated the optical- $\gamma$-ray correlation function for the entire prompt emission, and they found that for the contemporaneous rise at $0-18$ $\mathrm{s}$ and fall at $43-60 \mathrm{~s}$, the correlation coefficient has a maximum at lags between 0 and $5 \mathrm{~s}$, while for the middle part of the prompt emission, the correlation function still has a peak at lags between -1 to $3 \mathrm{~s}$, but is less prominent. To be conservative, we adopt the largest value $5 \mathrm{~s}$ as the arrival lag $\Delta t$ between the $\mathrm{MeV}$ photons and the optical photons. From eq.(9) we thus have for this burst

$$
\gamma_{\mathrm{eV}}-\gamma_{\mathrm{MeV}}<1.2 \times 10^{-7}
$$

\section{DISCUSSION}

In the last two sections, we have proposed a practical method for using cosmic transients for testing the energy dependence of the $\gamma$ parameter quantifying departures from the EEP, and we have taken GRBs as an example to illustrate the capabilities of this method. In the following, we discuss some of the uncertainties involved in this method and the caveats on our constraint results.

The largest uncertainty is from the intrinsic time delay between two test photons, $\Delta t_{\text {int }}$. Since we are only seeking upper limits, in the calculations we neglected the $\Delta t_{\text {int }}$ term based on the assumption that $\Delta t_{\text {int }}$ has the same sign as $\Delta t_{\text {obs }}$. More specifically, here we assumed that the $\mathrm{GeV}$ (optical) photons are intrinsically emitted later than (or at most simultaneously with) the MeV photons in GRB 090510 (GRB 080319B). From the observational point of view, such an assumption is empirically justified, since we have never observed any GRBs with $\mathrm{GeV}$ or optical photons arriving earlier than the $\mathrm{MeV}$ photons. In standard GRB models, the MeV photons are assumed to arise in internal shock regions via synchrotron emission, while $\mathrm{GeV}$ photons are usually believed to originate from the inverse Compton up-scattering of $\mathrm{MeV}$ photons, and the optical photons are usually assumed to arise in the external shock region (which is outer and occurs later than the internal shock) also via synchrotron emission. In other words, from the theoretical point of view, our assumption about the intrinsic order of $\mathrm{GeV}$ or optical photons with $\mathrm{MeV}$ photons is also standard practice. However, if in reality the $\mathrm{GeV}$ or optical photons are emitted earlier than the $\mathrm{MeV}$ photons, and $\left|\Delta t_{\text {int }}\right| \gg\left|\Delta t_{\text {obs }}\right|$, our constraint results would be invalidated.

The second uncertainty comes from the determination of $\Delta t_{\text {obs }}$. For GRB 090510, we chose $\Delta t_{\text {obs }}$ as the time delay between the $31-\mathrm{GeV}$ photon and the trigger photons for the Swift satellite. Our results could be more stringent if in reality the $31-\mathrm{GeV}$ photon were correlated with the other GBM (MeV) pulses, for example the seventh pulse which coincides in time with the $\mathrm{GeV}$ photon (Abdo et al. 2009). However, in principle, it would be conceivable that the $\mathrm{GeV}$ photon is correlated with some other $\mathrm{MeV}$ photons emitted earlier but which failed to trigger the Swift satellite. This would be similar to a case where intrinsically this $\mathrm{GeV}$ photon is emitted earlier than the $\mathrm{MeV}$ photons, which could potentially invalidate our results. For GRB 080319B, we adopted the time delay results from the optical- $\gamma$-ray correlation function. These results should be reliable, since the TORTORA telescope provided high time resolution measurements for the brightest portion of the optical flash. Furthermore, it is worth noting that our adopted delay time is even larger than the time delay between the onset times of the optical observations and the $\mathrm{MeV}$ trigger.

The total mass and the exact gravitational potential function of our galaxy is not well known. More accurate values for $M_{\mathrm{mw}}$ or for the function $U_{\mathrm{mw}}(r)$ could improve our constraints, but the correction should be limited to less than an order of magnitude.

In the calculations presented we neglect the time delay terms caused by the host galaxy and the intergalactic background gravitational potential. In principle, these terms could be much larger than $\Delta t_{\text {gra,mw }}$. With future observations, and with a better understanding of the potential functions for these terms, our result could be improved by orders of magnitude.

\section{CONCLUSION}

In principle, the accuracy of the EEP may be characterized by limits on the differences between the $\gamma$ values (one of the PPN parameters) for different species of particles, or for the same specie of particle at different energies. In this work, we propose that the time delays between correlated photons from cosmological transients, such as GRBs, can be used to constrain the accuracy of the EEP. Using the data from two observed GRBs as an example, and assuming that the observed time delays between photons of different energies are caused dominantly by the gravitational effects of our galaxy, an assumption which we justified in $\S 2$, for the $\mathrm{GeV}$ and $\mathrm{MeV}$ photons of one burst we give an upper limit of $\gamma_{\mathrm{GeV}}-\gamma_{\mathrm{MeV}}<2 \times 10^{-8}$; and for the optical and $\mathrm{MeV}$ photons form another burst we find $\gamma_{\mathrm{eV}}-\gamma_{\mathrm{MeV}}<1.2 \times 10^{-7}$.

Previous to this present work, the most accurate constraints on $\gamma$ differences were based on the photons and neutrinos from Supernova 1987A, giving an upper limit 
of $0.37 \%$ for optical photons and $\mathrm{MeV}$ neutrinos, and an upper limit of $1.6 \times 10^{-6}$ for two neutrinos, whose energy difference was of order $\sim \mathrm{MeV}$. If our present interpretation is correct, our results increase the accuracy level of such EEP constraints by at least one order of magnitude, to $\sim 10^{-7}$, while extending the energy range tested to the $\mathrm{GeV}-\mathrm{MeV}$ and $\mathrm{MeV}-\mathrm{eV}$ range.

A new type of transients, called Fast Radio Bursts, have lately attracted attention (Thornton et al. 2013). Although their physical origin is still debated (Kulkarni et al. 2014), if in the future they are proven to be cosmic transients, their simple sharp features make them attractive candidates for constraining the EEP, which could further extend the tested energy range to the radio band with high accuracy.

It is worth pointing out that high energy neutrinos have long been proposed to be associated with GRBs, although a dedicated search of high-energy neutrinos coincident with electromagnetically detected GRBs has so far led to null results (Abbasi et al. 2012; Aartsen et al. 2015). If in the future GRBs with both high energy photons and neutrinos are detected, better constraints on EEP might be achieved. On the other hand, neutrino detectors such as IceCube have reached the sensitivity to detect high-energy neutrinos of astrophysical origin for the first time. The three-year discovery catalog of IceCube contains a total of 37 neutrino candidate events with deposited energies ranging from 30 to $2000 \mathrm{TeV}$ (Aartsen et al. 2014). Although the origin of these neutrinos is still debated, some of them seem to have both spatial and temporal correlations, such as events 24 and 25 in the catalog (Aartsen et al. 2014). In the future, if the origin of these neutrinos is better understood, or the association between different detected events is confirmed, it will be essential to use these high energy cosmic neutrinos for obtaining better constraints on the accu- racy of the EEP.

The most precise previous constraints on the absolute $\gamma$ value of photons is $\gamma-1 \sim 10^{-5}$, which comes from radio photons. Our relative limits are three orders of magnitude lower than this, focusing on the value of the difference between various energies, rather than the absolute value. On the other hand, the Shapiro time-delay measurements using the Hipparcos optical astrometry satellite yielded an agreement with General Relativity of $\gamma_{e V}-1 \lesssim 0.3 \%$ (Froeschle et al. 1997). Based on our results, we can predict that the constraint on the absolute $\gamma$ values $\mathrm{MeV}$ or $\mathrm{GeV}$ photons, which from the sources we investigated here differ from that of their corresponding optical photons by $\lesssim 10^{-7}$, should agree with GR at least to this same $\lesssim 0.3 \%$ level of accuracy. Thus, we have extended the validity of this absolute value bound on $\gamma$ at this level from the optical to the $\mathrm{GeV}$ range, and have constrained the relative variation of $\gamma$ between optical and $\mathrm{GeV}$ to the $\lesssim 10^{-7}$ range.

We thank the anonymous referee for a valuable report and Derek Fox, Bing Zhang, Zi-Gao Dai, XiangYu Wang and Mou-Yuan Sun for helpful comments. This work is supported by National Basic Research Program ('973' Program) of China (grants 2014CB845800 and 2013CB834900), NASA NNX 13AH50G, the National Natural Science Foundation of China (grants nos. 11322328 and 11433009). XFW is partially supported by the One-Hundred-Talents Program, the Youth Innovation Promotion Association, and the Strategic Priority Research Program "The Emergence of Cosmological Structures" (grant no. XDB09000000) of of the Chinese Academy of Sciences, and the Natural Science Foundation of Jiangsu Province (grant no. BK2012890).

\section{REFERENCES}

Aartsen, M. G., Ackermann, M., Adams, J., et al. 2014, Physical Review Letters, 113, 101101

Aartsen, M. G., Ackermann, M., Adams, J., et al. 2015, ApJ, 805, L5

Abbasi, R., Abdou, Y., Abu-Zayyad, T., et al. 2012, Nature, 484, 351

Abdo, A. A., Ackermann, M., Ajello, M., et al. 2009, Nature, 462, 331

Amelino-Camelia, G., Ellis, J., Mavromatos, N. E., Nanopoulos, D. V., \& Sarkar, S. 1998, Nature, 393, 763

Amsler, C., Doser, M., Antonelli, M., et al. 2008, Physics Letters $\mathrm{B}, 667,1$

Atwood, W. B., Abdo, A. A., Ackermann, M., et al. 2009, ApJ, 697, 1071

Bertotti, B., Iess, L., \& Tortora, P. 2003, Nature, 425, 374

Bhat, P. N., Fishman, G. J., Meegan, C. A., et al. 1992, Nature, 359,217

Burrows, D. N., Hill, J. E., Nousek, J. A., et al. 2005, Space Sci. Rev., 120, 165

Ćwiok, M., Dominik, W., Małek, K., et al. 2007, Ap\&SS, 309, 531

Ellis, J., Mavromatos, N. E., Nanopoulos, D. V., Sakharov, A. S. \& Sarkisyan, E. K. G. 2006, Astroparticle Physics, 25, 402

Froeschle, M., Mignard, F., \& Arenou, F. 1997, Hipparcos - Venice '97, 402, 49

Gehrels, N., Chincarini, G., Giommi, P., et al. 2004, ApJ, 611, 1005

Gillessen, S., Eisenhauer, F., Trippe, S., et al. 2009, ApJ, 692, 1075

Guiriec, S., Connaughton, V., \& Briggs, M. 2009, GRB Coordinates Network, 9336, 1

Hoversten, E. A., Krimm, H. A., Grupe, D., et al. 2009, GCN Report, 218, 1
Kafle, P. R., Sharma, S., Lewis, G. F., \& Bland-Hawthorn, J. 2012 ApJ, 761, 98

Karpov, S., Beskin, G., Bondar, S., et al. 2008, GRB Coordinates Network, 7502, 1

Krauss, L. M., \& Tremaine, S. 1988, Physical Review Letters, 60, 176

Kulkarni, S. R., Ofek, E. O., Neill, J. D., Zheng, Z., \& Juric, M. 2014, arXiv:1402.4766

Kumar, P., \& Narayan, R. 2009, MNRAS, 395, 472

Kumar, P., \& Zhang, B. 2014, Phys. Rep., 561, 1

Lambert, S. B., \& Le Poncin-Lafitte, C. 2009, A\&A, 499, 331

Lambert, S. B., \& Le Poncin-Lafitte, C. 2011, A\&A, 529, A70

Longo, M. J. 1988, Physical Review Letters, 60, 173

McMillan, P. J. 2011, MNRAS, 414, 2446

Meegan, C., Lichti, G., Bhat, P. N., et al. 2009, ApJ, 702, 791

McBreen, S., Krühler, T., Rau, A., et al. 2010, A\&A, 516, A71

Molinari, E., Bondar, S., Karpov, S., et al. 2006, Nuovo Cimento B Serie, 121, 1525

Ohno, M., \& Pelassa, V. 2009, GRB Coordinates Network, 9334, 1

Pan, Y., Gong, Y., Cao, S., Gao, H., \& Zhu, Z.-H. 2015, arXiv:1505.06563

Racusin, J. L., Karpov, S. V., Sokolowski, M., et al. 2008a, Nature, 455, 183

Racusin, J. L., Gehrels, N., Holland, S. T., et al. 2008b, GRB Coordinates Network, 7427, 1

Rau, A., McBreen, S., \& Kruehler, T. 2009, GRB Coordinates Network, 9353, 1

Roming, P. W. A., Kennedy, T. E., Mason, K. O., et al. 2005, Space Sci. Rev., 120, 95

Shapiro, I. I. 1964, Physical Review Letters, 13, 789 
Thornton, D., Stappers, B., Bailes, M., et al. 2013, Science, 341, 53

Vreeswijk, P. M., Milvang-Jensen, B., Smette, A., et al. 2008, GRB Coordinates Network, 7451, 1

Wiklind, T., Mobasher, B., \& Bromm, V. 2013, Astrophysics and Space Science Library, 396,

Will, C. M. 1993, Theory and Experiment in Gravitational Physics, by Clifford M. Will, pp. 396. ISBN 0521439736. Cambridge, UK: Cambridge University Press, March 1993.,
Will, C. M. 2006, Living Reviews in Relativity, 9, 3 Will, C. M. 2014, Living Reviews in Relativity, 17, 4 Zou, Y.-C., Piran, T., \& Sari, R. 2009, ApJ, 692, L92 